\title{
On the Lipschitz regularity of solutions of a minimum problem with free boundary
}

\author{
ARAM L. KARAKHANYAN ${ }^{\dagger}$ \\ Centre for Mathematics and Its Applications, Australian National University, \\ Canberra, ACT 0200, Australia \\ Current address: Department of Mathematics, University of Texas at Austin, \\ 1 University Station C1200, Austin, TX 78712, USA
}

[Received 8 May 2006 and in revised form 1 September 2007]

In this article under the assumption of "small" density for the negativity set, we prove local Lipschitz regularity for the two-phase minimization problem with free boundary for the functional

$$
\mathcal{E}_{p}(v, \Omega)=\int_{\Omega}\left[|\nabla v|^{p}+\lambda_{1}^{p} \chi_{\{u \leqslant 0\}}+\lambda_{2}^{p} \chi_{\{u>0\}}\right], \quad 1<p<\infty,
$$

where $\lambda_{1}, \lambda_{2}$ are positive constants so that $\Lambda=\lambda_{1}^{p}-\lambda_{2}^{p}<0, \chi_{D}$ is the characteristic function of the set $D, \Omega \subset \mathbb{R}^{n}$ is a (smooth) domain and the minimum is taken over a suitable subspace of $W^{1, p}(\Omega)$.

\section{Introduction}

Let $\mathcal{K}_{g}=\left\{v \in W^{1, p}(\Omega): v-g \in W_{0}^{1, p}(\Omega)\right\}$ for a given smooth function $g$ on $\Omega \subset \mathbb{R}^{n}$ and consider the energy minimization problem

$$
\mathcal{E}_{p}(u, \Omega)=\inf _{v \in \mathcal{K}_{g}} \mathcal{E}_{p}(v, \Omega), \quad 1<p<\infty
$$

with

$$
\mathcal{E}_{p}(u, \Omega)=\int_{\Omega}\left[|\nabla u|^{p}+\lambda_{1}^{p} \chi_{\{u \leqslant 0\}}+\lambda_{2}^{p} \chi_{\{u>0\}}\right] .
$$

Here $\Omega \subset \mathbb{R}^{n}$ is a bounded and smooth domain, $\lambda_{1}, \lambda_{2}$ are positive constants with $\Lambda=\lambda_{1}^{p}-\lambda_{2}^{p}<0$, and $\chi_{M}$ is the characteristic function of the set $M \subset \mathbb{R}^{n}$, i.e.

$$
\chi_{M}= \begin{cases}1 & \text { if } x \in M, \\ 0 & \text { if } x \notin M .\end{cases}
$$

The minimizer $u$ is expected to satisfy the following overdetermined problem:

$$
\begin{aligned}
& \Delta_{p} u=0 \quad \text { in } u \neq 0, \\
& \left|\nabla u^{+}\right|^{p}-\left|\nabla u^{-}\right|^{p}=c \quad \text { on } \partial\{u>0\}, \quad u=g \quad \text { on } \partial \Omega,
\end{aligned}
$$

\footnotetext{
†E-mail: aram.karakhanyan@maths.anu.edu.au, aram@math.utexas.edu
} 
where the $u^{+}, u^{-}$are respectively the positive and negative parts of $u, c$ is a positive constant and the boundary data $g$ is not necessarily nonnegative. This problem, usually termed a Bernoullitype problem, models for example cavitational flow of one or two perfect fluids, or equilibrium configuration for heat or electrostatic energy optimization. Weak solutions of problem (2) can be obtained by minimizing $\mathcal{E}_{p}$ (see Theorem 2 ), and our objective here is to analyze the regularity of those solutions $u$.

Since $u$ has a jump along the free boundary $\Gamma=\partial\{u>0\}$, the best expected regularity for $u$ is Lipschitz continuity. In the classical case $p=2$, corresponding to the usual Laplacian, this is proved in $[\mathrm{ACF}]$, and in $[\overline{\mathrm{DP}}]$ for any $1<p<\infty$ and $u^{-} \equiv 0$. The main difficulty in attacking the Lipschitz regularity for the general case is the lack of monotonicity formulas, firstly introduced in $[\mathrm{ACF}]$, and subsequently developed in $[\overline{\mathrm{CJK}}],[\mathrm{CKS}]$. However, we can still prove that $u \in C_{\text {loc }}^{0,1}$ if the negativity set $\Omega^{-}(u)=\{u<0\}$ is reasonably small. The $C^{0,1}$ estimate plays a vital role in establishing $C^{1, \alpha}$ regularity of the free boundary near flat points. However, here we solely focus upon proving a local $C^{0,1}$ estimate for solutions. The present study has been inspired by a recent work $[\overline{\mathrm{KKS}}]$ and by $[\overline{\mathrm{LS}}]$, where a similar result is proven for another overdetermined problem:

$$
F\left(D^{2} u\right)=\chi\{D\} \quad \text { in } B_{1}, \quad u=|\nabla u|=0 \quad \text { in } B_{1} \backslash D,
$$

for a certain class of uniformly elliptic operators $F$. We observe here that in contrast to (3) we do not have a pde which would be satisfied by solutions $u$ of $(1)$ in $\Omega$.

\section{Preliminaries}

The following notations are used throughout the paper: $\Omega \subset \mathbb{R}^{n}$ is a smooth and bounded domain, $g$ is a smooth function defined on some neighborhood of $\partial \Omega, W^{1, p}(\Omega), W_{0}^{1, p}(\Omega)$ are the usual Sobolev spaces, $B_{R}(y)=\left\{x \in \mathbb{R}^{n}:|x-y|<R\right\}, B_{R}=B_{R}(0), u^{ \pm}$are respectively the positive and negative parts of $u, \chi_{D}$ the characteristic function of $D$, and $\Gamma=\partial\{u>0\}$ the free boundary. Let $\lambda_{1}$ and $\lambda_{2}$ be two positive constants so that $\Lambda=\lambda_{1}^{p}-\lambda_{2}^{p}<0$ where $1<p<\infty$. Consider the functional

$$
\mathcal{E}_{p}(u, \Omega)=\int_{\Omega}\left[|\nabla u|^{p}+\lambda_{1}^{p} \chi_{\{u \leqslant 0\}}+\lambda_{2}^{p} \chi_{\{u>0\}}\right] .
$$

In what follows we denote by $\lambda(u)$ the following function:

$$
\lambda(u)= \begin{cases}\lambda_{1}^{p} & \text { if } u \leqslant 0 \\ \lambda_{2}^{p} & \text { if } u>0\end{cases}
$$

As in the classical paper [ACF] we define $\lambda(0)=\lambda_{1}^{p}$ if $\Lambda<0$ and $\lambda(0)=\lambda_{2}^{p}$ if $\Lambda>0$. For brevity we focus on the case $\Lambda<0$. Existence of solutions to (1) easily follows from the lower semicontinuity of $\mathcal{E}_{p}$ as in $\mathrm{ACF}$.

THEOREM 1 Let $u$ be a (local) minimizer of $\mathcal{E}_{p}$. Then $u$ is bounded.

Proof. First observe that

$$
\int_{\Omega}\left[|\nabla u|^{p}+\lambda_{1}^{p} \chi_{\{u \leqslant 0\}}+\lambda_{2}^{p} \chi_{\{u>0\}}\right]=\int_{\Omega}\left[|\nabla u|^{p}+\Lambda \chi_{\{u \leqslant 0\}}\right]+\lambda_{2}^{p} \text { meas } \Omega .
$$


For given $D \subset \Omega$ consider the functional $I_{0}(u, D)=\int_{D}\left[|\nabla u|^{p}+\Lambda \chi_{\{u \leqslant 0\}}\right]$. If $u$ is a minimizer of $\mathcal{E}_{p}(u, D)$ then it is also a minimizer of $I_{0}(u, D)$ and vice versa since the difference between $I_{0}$ and $\mathcal{E}_{p}$ is a constant for given $D$.

Now take $u_{\varepsilon}=u+\varepsilon \min (M-u, 0)$, where $M=\sup g>0$ and $\varepsilon$ is a small positive number. Then taking $D=\Omega$ and testing $u$ against $u_{\varepsilon}$ we get

$$
\int_{\Omega}\left[|\nabla u|^{p}+\Lambda \chi_{\{u \leqslant 0\}}\right] \leqslant \int_{\Omega}\left[\left|\nabla u_{\varepsilon}\right|^{p}+\Lambda \chi_{\left\{u_{\varepsilon} \leqslant 0\right\}}\right] .
$$

Note that $u$ and $u_{\varepsilon}$ are different on the set $\{u>M\}$, therefore the last inequality becomes

$$
\int_{\Omega \cap\{u>M\}}|\nabla u|^{p} \leqslant \int_{\Omega \cap\{u>M\}}\left[|\nabla u|^{p}(1-\varepsilon)^{p}+\Lambda \chi_{\left\{u_{\varepsilon} \leqslant 0\right\}}\right],
$$

which is a contradiction since $\Lambda<0$ and hence $u \leqslant M$. Now take $u_{\varepsilon}=u-\min (u-m, 0)$ where $m=\inf g<0$ and $\varepsilon$ is a positive number. Again since $u$ is a minimizer we have

$$
\int_{\Omega}\left[|\nabla u|^{p}+\Lambda \chi_{\{u \leqslant 0\}}\right] \leqslant \int_{\Omega}\left[\left|\nabla u_{\varepsilon}\right|^{p}+\Lambda \chi_{\left\{u_{\varepsilon} \leqslant 0\right\}}\right] .
$$

On the set $\{u<m\}$, where $u$ and $u_{\varepsilon}$ are different, we have

$$
\int_{\Omega \cap\{u<m\}}\left[|\nabla u|^{p}+\Lambda \chi_{\{u<m\}}\right] \leqslant \int_{\Omega \cap\{u<m\}}\left[\left|\nabla u_{\varepsilon}\right|^{p}(1-\varepsilon)^{p}+\Lambda \chi_{\left\{u \leqslant-\frac{\varepsilon m}{1-\varepsilon}\right\}}\right] .
$$

Note that $-\frac{\varepsilon m}{1-\varepsilon}>0$ and therefore

$$
\begin{aligned}
\int_{\Omega \cap\{u<m\}}|\nabla u|^{p} & \leqslant \int_{\Omega \cap\{u<m\}}\left[\left|\nabla u_{\varepsilon}\right|^{p}(1-\varepsilon)^{p}+\Lambda\right]\left(\chi_{\left\{u \leqslant-\frac{\varepsilon m}{1-\varepsilon}\right\}}-\chi_{\{u<m\}}\right) \\
& =\int_{\Omega \cap\{u<m\}}\left|\nabla u_{\varepsilon}\right|^{p}(1-\varepsilon)^{p} .
\end{aligned}
$$

This implies that $m \leqslant u$.

THEOREM $2 u \in C_{\mathrm{loc}}^{\alpha}(\Omega)$.

Proof. Let $B_{R}(y) \subset \Omega$ and $w$ be the solution to the following Dirichlet problem:

$$
\Delta_{p} w=0 \quad \text { in } B_{R}(y), \quad w=u \quad \text { on } \partial B_{R}(y) .
$$

Then

$$
\int_{B_{R}(y)}\left[|\nabla u|^{p}+\lambda(u)\right] \leqslant \int_{B_{R}(y)}\left[|\nabla w|^{p}+\lambda(w)\right]
$$

where $\lambda(u)=\lambda_{1}^{p} \chi_{\{u \leqslant 0\}}+\lambda_{2}^{p} \chi_{\{u>0\}}$. Note that we also have

$$
\int_{B_{R}(y)}|\nabla u|^{p} \geqslant \int_{B_{R}(y)}|\nabla w|^{p} .
$$


Since $\lambda(u)$ is bounded this implies that

$$
\int_{B_{R}(y)}\left[|\nabla u(x)|^{p}-|\nabla v(x)|^{p}\right] \mathrm{d} x \leqslant C R^{n}
$$

Furthermore, from $[\overline{\mathrm{DP}}]$ one has

$$
\begin{aligned}
\int_{B_{R}(y)}\left[|\nabla u|^{p}-|\nabla v|^{p}\right] & \\
\geqslant & \begin{cases}c\left(\int_{B_{R}(y)}|\nabla(u-v)|^{p}\right)^{2 / p}\left(\int_{B_{R}(y)}|\nabla u|^{p}\right)^{1-2 / p}, & 1<p \leqslant 2, \\
c \int_{B_{R}(y)}|\nabla(u-v)|^{p}, & 2 \leqslant p<\infty .\end{cases}
\end{aligned}
$$

which together with $(6)$ implies that

$$
\int_{B_{R}(y)}|\nabla(u-w)|^{p} \leqslant \begin{cases}C \lambda_{+}^{p^{2} / 2} R^{n p / 2}\left(\int_{B_{R}(y)}|\nabla u|^{p}\right)^{1-p / 2}, & 1<p \leqslant 2, \\ C \lambda_{+}^{p} R^{n}, & 2 \leqslant p<\infty .\end{cases}
$$

Recall that from the gradient estimates for harmonic functions we have

$$
\sup _{B_{R / 2}(y)}|\nabla w| \leqslant C \frac{\sup _{\Omega}|u|}{R} .
$$

Now for small $R$ and $p>2$ we have

$$
\begin{aligned}
\int_{B_{R / 2}(y)}|\nabla u|^{2} & \leqslant C \int_{B_{R / 2}(y)}|\nabla(u-w)|^{p}+C \int_{B_{R / 2}(y)}|\nabla w|^{p} \\
& \leqslant C \int_{B_{R / 2}(y)}|\nabla(u-w)|^{p}+C R^{n-p} .
\end{aligned}
$$

Then combining (8) and (9) as in [DP] the result follows.

COROLlaRY $1 u$ is $p$-subharmonic.

Proof. We first note that if $v$ satisfies

$$
\Delta_{p} v=0 \quad \text { in } B_{R}(y), \quad v=u \quad \text { on } \partial B_{R}(y),
$$

where $B_{R}(y) \subset \Omega$, then testing $u$ against $\min (u, v)$ we find that

$$
\int_{B_{R}(y)}\left[|\nabla u(x)|^{p}-|\nabla \min (u(x), v(x))|^{p}\right] \mathrm{d} x \leqslant \Lambda \int_{B_{R} \cap\{u>0 \geqslant v\}} 1 \mathrm{~d} x .
$$

Since $u$ is Hölder continuous, the set $\{u>v\}$ is open and we can apply (7) to infer that

$$
\int_{B_{R}(y)}\left[|\nabla u|^{p}-|\nabla \min (u, v)|^{p}\right]>0 .
$$

However, $\Lambda<0$, which yields $\max (u-v, 0)=0$ in $B_{R}$, that is, $u \leqslant v$ in $B_{R}$. Hence $u$ is $p$-subharmonic in $\Omega$. 
Before proceeding further we summarize some basic properties of solutions to (1).

THEOREM 3 Let $u$ be the solution to (1). Then

- $\Delta_{p} u=0$ in $[\{u>0\} \cup\{u<0\}] \cap \Omega$,

- $\Delta_{p} u \geqslant 0$ in $\Omega$,

- $\lim _{\varepsilon \downarrow 0} \int_{\partial\{u<-\varepsilon\}}\left((p-1)|\nabla u|^{p}-\lambda_{1}^{p}\right) v \cdot \eta+\lim _{\delta \downarrow 0} \int_{\partial\{u>\delta\}}\left((p-1)|\nabla u|^{p}-\lambda_{2}^{p}\right) v \cdot \eta=0$

for any $\eta \in C_{0}^{1}\left(\Omega, \mathbb{R}^{n}\right)$ provided meas $\{u=0\}=0$.

The proof follows precisely as in $[\mathrm{ACF}$.

\section{Main result}

In this section we assume that $\lambda_{1}=0$, since introducing $\lambda_{0}^{p}=\lambda_{2}^{p}-\lambda_{1}^{p}=-\Lambda>0$ we can consider a new functional

$$
\int_{\Omega}\left[|\nabla u|^{p}+\lambda_{0}^{p} \chi_{\{u>0\}}\right]=\mathcal{E}_{p}(u, \Omega)-\lambda_{1}^{p} \text { meas } \Omega .
$$

Therefore we identify $\mathcal{E}_{p}(u, \Omega)$ with $\int_{\Omega}\left[|\nabla u|^{p}+\lambda^{p} \chi_{\{u>0\}}\right]$ for some positive constant $\lambda$. Next we define the main class of functions that we are going to work with.

DEFINITION 1 Let $z$ be a fixed point and $0<r<1$. Then $u$ is said to be of class $\mathcal{Q}_{r}(z, M)$ if

(i) $u$ is a local minimizer of $\mathcal{E}_{p}$ in $B_{r}(z)$,

(ii) $\sup _{B_{r}(z)}|u| \leqslant M$,

(iii) $z \in \partial\{u>0\}$.

Let

$$
\Theta\left(x_{0}, r\right)=\frac{\operatorname{meas}\left(\{u<0\} \cap B_{r}\right)}{\operatorname{meas} B_{r}}, \quad x_{0} \in \partial\{u>0\} .
$$

THEOREM 4 Let $u \in \mathcal{Q}_{1}\left(x_{0}, M\right)$. There exists a universal constant $C>0$ such that

$$
|u(x)| \leqslant \frac{2 M}{C}|x|
$$

provided $\Theta\left(x_{0}, r\right) \leqslant C$ for all $0<r<1$.

Proof. Without loss of generality we may assume $x_{0}=0$. It is enough to prove that

$$
\sup _{B_{2}-(k+1)}|u(x)| \leqslant \max \left\{\frac{M}{C 2^{k}}, \frac{S(k)}{2}, \ldots, \frac{S(k-m)}{2^{m+1}}, \ldots, \frac{S(0)}{2^{k+1}}\right\}
$$

where $S(k)=\sup _{B_{2-k}}|u|$. Assume otherwise. Then there are integers $k_{j}, j=1,2, \ldots$, so that

$$
\sup _{B_{2}-\left(k_{j}+1\right)}\left|u_{j}(x)\right|>\max \left\{\frac{j M}{2^{k_{j}}}, \frac{S_{j}\left(k_{j}\right)}{2}, \ldots, \frac{S_{j}\left(k_{j}-m\right)}{2^{m+1}}, \ldots, \frac{S_{j}(0)}{2^{k_{j}+1}}\right\}
$$

and

$$
\Theta\left(0,2^{-k_{j}}\right) \leqslant 1 / j \rightarrow 0
$$


Here

$$
S_{j}\left(k_{j}-m\right)=\sup _{B_{2}-\left(k_{j}-m\right)}\left|u_{j}\right|, \quad m=0,1, \ldots, k_{j},
$$

and $u_{j} \in \mathcal{Q}_{1}(z, M)$. Observe that $\left|u_{j}\right| \leqslant M$ implies $k_{j} \rightarrow \infty$.

Consider the auxiliary function $v_{j}$ defined as

$$
v_{j}(x)=\frac{u_{j}\left(x 2^{-k_{j}}\right)}{S_{j}\left(k_{j}+1\right)} .
$$

We start by proving $W^{1, p}$ estimates for $v_{j}$. Set $\sigma_{j}=2^{-k_{j}} S_{j}^{-1}\left(k_{j}+1\right)$. Note that by $11, \sigma_{j} \leqslant$ $j^{-1} \rightarrow 0$. For fixed $R_{0}>0$ we have

$$
\int_{B_{R_{0}}}\left|\nabla v_{j}(x)\right|^{p} \mathrm{~d} x=\sigma_{j}^{p} \int_{B_{R_{0}}}\left|\nabla u_{j}\left(x 2^{-k_{j}}\right)\right|^{p} \mathrm{~d} x=\sigma_{j}^{p} 2^{n k_{j}} \int_{B_{R_{0} 2^{-k_{j}}}}\left|\nabla u_{j}(y)\right|^{p} \mathrm{~d} y .
$$

Let $\rho>0$ and $\varphi$ be the standard cut-off function of $B_{\rho}$. Then $\eta=\varphi^{p} u_{j}^{+}$is an admissible test function and (ii) yields

$$
\int_{B_{\rho}}\left|\nabla u_{j}^{+}\right|^{p-2} \nabla u_{j}^{+} \nabla \eta \leqslant 0 .
$$

Rearranging terms and using the Hölder inequality we get

$$
\begin{aligned}
\int_{B_{\rho}} \varphi^{p}\left|\nabla u_{j}^{+}\right|^{p} & \leqslant p \int_{B_{\rho}}\left|\nabla u_{j}^{+}\right|^{p-1} \varphi^{p-1}|\nabla \varphi| u_{j}^{+} \\
& \leqslant p\left(\int_{B_{\rho}}|\nabla \varphi|^{p}\left(u_{j}^{+}\right)^{p}\right)^{1 / p}\left(\int_{B_{\rho}}\left|\nabla u_{j}^{+}\right|^{p} \varphi^{p}\right)^{1-1 / p} .
\end{aligned}
$$

So we get Caccioppoli's inequality

$$
\int_{B_{\rho / 2}}\left|\nabla u_{j}^{+}\right|^{p} \leqslant \frac{c}{\rho^{p}} \int_{B_{\rho}}\left(u_{j}^{+}\right)^{p} \leqslant c \rho^{n-p}\left(\sup _{B_{\rho}}\left|u_{j}\right|\right)^{p} .
$$

Let us take $\rho / 2=R_{0} / 2^{k_{j}}$ in the last inequality,

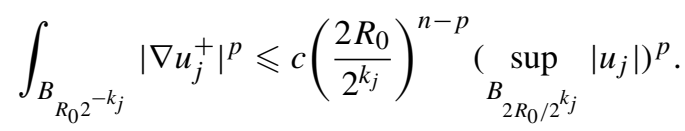

Choose $R_{0}=2^{l-1}$ for a fixed integer $l<k_{j}$. Then

$$
\begin{aligned}
\int_{2^{l-1}}\left|\nabla v_{j}^{+}\right|^{p} & \leqslant c\left[\frac{2^{-k_{j}}}{S_{j}\left(k_{j}+1\right)}\right]^{p} 2^{n k_{j}} 2^{\left(l-k_{j}\right)(n-p)}\left(\sup _{2^{l-k_{j}}}\left|u_{j}\right|\right)^{p} \\
& \leqslant c\left[\frac{2^{-k_{j}}}{S_{j}\left(k_{j}+1\right)}\right]^{p} 2^{n k_{j}} 2^{\left(l-k_{j}\right)(n-p)}\left(2^{l+1} S_{j}\left(k_{j}+1\right)\right)^{p}=2^{l n+p},
\end{aligned}
$$


where the second inequality follows from (11). Therefore $\left\|\nabla v_{j}\right\|_{L^{p}}$ is locally bounded, implying local uniform $W^{1, p}$ estimates for $v_{j}$ for $j$ large.

If $p>n$ then the Sobolev imbedding theorem implies uniform local $C^{\alpha}$ estimate for $v_{j}$, for $j$ large. Suppose $1<p \leqslant n$. Consider the scaled energy functional

$$
\mathcal{E}_{j}(v, D)=\int_{D}\left[|\nabla v|^{p}+\sigma_{j}^{p} \lambda^{p} \chi_{\{v>0\}}\right] .
$$

First observe that a simple calculation gives

$$
\mathcal{E}_{j}\left(v_{j}, B_{R_{0}}\right)=\sigma_{j}^{p} 2^{n k_{j}} \mathcal{E}_{p}\left(u_{j}, B_{R_{0} 2^{-k_{j}}}\right) .
$$

Therefore $v_{j}$ is a solution to

$$
\mathcal{E}_{j}\left(v_{j}\right)=\inf _{v \in \mathcal{K}_{j}} \mathcal{E}_{j}(v), \quad \mathcal{K}_{j}=\left\{v \in W^{1, p}\left(B_{2^{k_{j}}}\right): v-v_{j} \in W_{0}^{1, p}\left(B_{2^{k_{j}}}\right)\right\}
$$

Applying Theorem 1 to $v_{j}$ we have a uniform $C_{\mathrm{loc}}^{\alpha}$ estimate. Using the uniform $W^{1, p}$ and $C_{\mathrm{loc}}^{\alpha}$ estimates we have, at least for a subsequence,

$$
v_{j} \rightarrow v_{\infty} \quad \text { in } W^{1, p}\left(B_{2}\right) \cap C^{\alpha}\left(B_{2}\right) .
$$

Now we claim that $v_{\infty}$ is a local minimizer of $D_{p}(v)=\int|\nabla v|^{p}$. Indeed, for any $\varphi \in C_{0}^{\infty}\left(B_{1}\right)$,

$$
\int_{B_{1}}\left[\left|\nabla v_{j}\right|^{p}+\sigma_{j}^{p} \lambda^{p} \chi_{\left\{v_{j}>0\right\}}\right] \leqslant \int_{B_{1}}\left[\left|\nabla\left(v_{j}+\varphi\right)\right|^{p}+\sigma_{j}^{p} \lambda^{p} \chi_{\left\{v_{j}+\varphi>0\right\}}\right] .
$$

By (19) we have

$$
\int_{B_{1}}\left|\nabla v_{j}\right|^{p} \rightarrow \int_{B_{1}}\left|\nabla v_{\infty}\right|^{p}, \quad \int_{B_{1}}\left|\nabla\left(v_{j}+\varphi\right)\right|^{p} \rightarrow \int_{B_{1}}\left|\nabla\left(v_{\infty}+\varphi\right)\right|^{p} .
$$

Since also $\sigma_{j} \leqslant 1 / j$, we get

$$
\sigma_{j}^{p} \int_{B_{1}} \lambda^{p} \chi_{\left\{v_{j}>0\right\}} \rightarrow 0, \quad \sigma_{j}^{p} \int_{B_{1}} \lambda^{p} \chi_{\left\{v_{j}+\varphi>0\right\}} \rightarrow 0 .
$$

Hence we conclude that

$$
\int_{B_{1}}\left|\nabla v_{\infty}\right|^{p} \leqslant \int_{B_{1}}\left|\nabla\left(v_{\infty}+\varphi\right)\right|^{p} .
$$

In view of $C^{\alpha}$ regularity this shows that $v_{\infty}$ is a local minimizer for $D_{p}(v)$ in $B_{1}$.

From the definition of $v_{j}$ and $(12)$ we conclude:

- $0 \leqslant v_{\infty} \leqslant 2$ in $B_{1}$,

- $\Delta_{p} v_{\infty}=0$ in $B_{1}$,

- $v_{\infty}(0)=0$,

- $\sup _{B_{1 / 2}}\left|v_{\infty}\right|=1$.

which contradicts the strong maximum principle. 
Corollary 2 Assume that $\Theta_{r}(z, r) \leqslant C$ for all $z \in B_{1 / 2} \cap \Gamma$. Then $u \in \mathcal{Q}_{1}(0, M)$ is Lipschitz in $B_{1 / 4}$.

Proof. Let $u(x)>0$ and $d(x)=\operatorname{dist}(x, \partial\{u>0\})$. Let $z \in \partial\{u>0\}$ so that $d(x)=|x-z|$. Then $u(x) \leqslant 2 M C^{-1} d(x)$. By Harnack's inequality $u \leqslant 2 c M C^{-1} d(x)$ in $B_{d(x) / 2}$. Consider $v(y)=$ $u(x+d(x) y) / d(x)$. Then

$$
\Delta_{p} v=0 \quad \text { in } B_{1}, \quad 0 \leqslant v(y) \leqslant 2 c M C^{-1} \quad \text { in } B_{1 / 2} .
$$

Then from the local gradient estimate $|\nabla v(0)| \leqslant C(n, p, M, C)$.

\section{REFERENCES}

[ACF] Alt, H. W., CAfFarelli, L. A., \& Friedman, A. Variational problems with two phases and their free boundaries. Trans. Amer. Math. Soc. 282 (1984), 431-461. Zbl $0844.35137 \mid$ MR 0732100

[CJK] Caffarelli, L. A., Jerison, D., \& Kenig, C. E. Some new monotonicity theorems with applications to free boundary problems. Ann. of Math. 155 (2002), 369-404. Zbl pre01779738 MR 1906591

[CKS] Caffarelli, L. A., Karp, L., \& Shahgholian, H. Regularity of a free boundary with application to the Pompeiu problem. Ann. of Math. 151 (2000), 269-292. Zbl 0960.35112 MR 1745013

[DP] Danielli, D., \& Petrosyan, A. A minimum problem with free boundary for a degenerate quasilinear operator. Calc. Var. Partial Differential Equations 23 (2005), 97-124. Zbl 1068.35187 MR 2133664

[KKS] Karakhanyan, A. L., Kenig, C. E., \& Shahgholian, H. The behavior of the free boundary near the fixed boundary for a minimization problem. Part II: Non-tangential touch. Manuscript.

[LS] Lee, K., \& Shahgholian, H. Regularity of a free boundary for viscosity solutions of nonlinear elliptic equations. Comm. Pure Appl. Math. 54 (2001), 43-56. Zbl 1020.35123 MR 1787106

[MZ] MALÝ, J., \& ZIEMER, W. P. Fine Regularity of Solutions of Elliptic Partial Differential Equations. \begin{tabular}{l|l|l|} 
Math. Surveys Monogr. 51, Amer. Math. Soc., Providence, RI (1997). Zbl 0882.35001 & MR 1461542 \\
\hline
\end{tabular} 